\title{
Alemtuzumab-induced cytokine release syndrome: an under-appreciated risk in treatment of delayed renal allograft function
}

\author{
Roberts DJ ${ }^{1}$, Olyaei $\mathrm{A}^{1,2}$, Rehman $\mathrm{S}^{1}$, Norman DJ ${ }^{1,3}$, de Mattos $\mathrm{AM}^{1}$ and Lockridge JB ${ }^{1 *}$ \\ ${ }^{1}$ Oregon Health \& Science University, Division of Nephrology \& Hypertension, Transplant Medicine Section, USA \\ ${ }^{2}$ Oregon State University, School of Pharmacy, Department of Pharmacy Practice, USA \\ ${ }^{3}$ Oregon Health \& Science University, Laboratory of Immunogenetics and Transplantation, USA
}

\begin{abstract}
Background: Alemtuzumab, an antilymphocyte (anti-CD52) monoclonal antibody, has been shown to be at least equivalent in efficacy compared to standard induction therapy in preventing acute rejection at one year post-transplantation, but is associated with three major categories of risk: increased susceptibility to infection, increased risk of lymphoproliferative malignancy, as well as a more acute risk of infusion-related reaction that can range widely in severity from mild fever to full cytokine release syndrome that is more likely to occur during the first dose. Alemtuzumab is more typically chosen for induction in high immunologic risk kidney recipients and in cases of delayed graft function at some transplant centers. However, its safety when used for delayed graft function has not been directly compared to other immunosuppressive agents, and the incidence of cytokine release syndrome from alemtuzumab or other lymphocyte-depleting agents is not known.
\end{abstract}

Case Presentation: We present two cases of flash pulmonary edema due to cytokine release syndrome in two kidney transplant recipients who received alemtuzumab for delayed graft function. In one case, dialysis was performed prior to alemtuzumab administration, while in the other case, euvolemia was already established by dialysis prior to the drug administration.

Conclusion: Cytokine release syndrome is an underappreciated risk from alemtuzumab. In our discussion, we illuminate the risks and propose preventive measures.

\begin{abstract}
Abbreviations: ESRD: End Stage Renal Disease; NODAT: New Onset Diabetes After Transplantation; KDPI: Kidney Donor Profile Index; DBD: Donation after Brain Death; DCD: Donation after Cardiac Death; SCD: Standard Criteria Donor; HLA: Human Leukocyte Antigen; PRA: Panel Reactive Antibodies; cPRA: Calculated Panel Reactive Antibodies
\end{abstract}

\section{Introduction}

Alemtuzumab is an antilymphocyte (anti-CD52) monoclonal antibody that exerts its therapeutic effects via $\mathrm{T}$-cell suppression and depletion [1]. While it has never been FDA-approved specifically for kidney transplantation, it has been shown to be at least equivalent in efficacy compared to standard induction therapy (basiliximab or rabbit antithymocyte globulin) in preventing acute rejection at one year posttransplantation $[2,3]$. As with all lymphocyte-depleting therapies, there are three major categories of risk from alemtuzumab. These risks are: increased susceptibility to infection, via its prolonged and potentially profound resultant lymphopenia, increased risk of lymphoproliferative malignancy, as well as a more acute risk of infusion-related reaction that can range widely in severity from mild fever to full cytokine release syndrome that is more likely to occur during the first dose $[1,3,4]$. The lymphopenic effect is its most commonly reported risk and has limited it from being routinely used in lower immunogenic risk recipients without strong indications over standard therapy [14]. Rather, alemtuzumab is more typically chosen for induction in high immunologic risk kidney recipients and in cases of delayed graft function at some transplant centers. While its safety may be equivalent to rabbit antithymocyte when given in the operating room at the time of transplantation, its safety when used for delayed graft function (i.e. not in the operating room while intubated and under the observation of an anesthesiologist) has not been directly compared to other immunosuppressive agents [3]. In addition, the incidence of cytokine release syndrome from alemtuzumab or other lymphocyte-depleting agents is not known. We present two cases of flash pulmonary edema due to cytokine release syndrome in two kidney transplant recipients who received alemtuzumab for delayed graft function. In our discussion, we illuminate the risks of cytokine release syndrome from alemtuzumab and propose preventive measures.

\section{Case Presentation}

\section{Case \# 1}

Our first patient in this discussion is a 57 -year-old Caucasian woman with ESRD due to medullary cystic kidney disease, who had relevant medical comorbidities including essential hypertension, peripheral vascular disease (including iliac involvement evident on preoperative imaging), and diabetes mellitus (controlled to an

${ }^{\star}$ Correspondence to: Joseph B Lockridge, MD, Oregon Health \& Science University, Division of Nephrology \& Hypertension, Transplant Medicine Section, USA, E-mail: lockridg@ohsu.edu

Key words: Kidney transplant, alemtuzumab, cytokine release syndrome, pulmonary edema

Received: September 14, 2020; Accepted: September 21, 2020; Published: September 24, 2020 
HbA1C of $7 \%$ on subcutaneous insulin). She had 2 prior deceased donor kidney transplants, the first in 1989 (failed in 1990), the second in 1998 (failed in 2015 with tubular atrophy and interstitial fibrosis), with the aforementioned diabetes classified as new onset after transplant (NODAT). Her surgical history was also notable for bilateral native nephrectomies (performed due to recurrent infections and nephrolithiasis prior to her first transplant) and splenectomy in 1982. She was on hemodialysis prior to presenting for her third deceased donor kidney transplant, with her final dialysis performed within the day prior to the transplantation course we discuss here. The kidney donor for this third transplant was 40 years old, had a profile index (KDPI) of 73\%, was a standard criteria (SCD), brain dead (DBD), donor who died due to a cerebrovascular event. There were 2 HLA-DR and zero HLA-A and HLA-B mismatches. Panel reactive antibodies (PRA) by flow cytometry were $16 \%$ for Class -I and $9 \%$ for Class -II. Calculated panel reactive antibody level (cPRA) was $91 \%$.

She underwent immunosuppressive induction in the operating room with $20 \mathrm{mg}$ of IV basiliximab given one hour following $500 \mathrm{mg}$ of IV methylprednisolone (which incidentally was $9.6 \mathrm{mg} / \mathrm{kg}$ in this particular patient). Kidney transplantation surgery was performed without any apparent immediate complications. Her induction with basiliximab was chosen by our center-specific protocol (determined to be low immunogenic risk, based on PRA $<50 \%$, no positive flow crossmatch, no blood type incompatibility, no prior delayed graft function, not African American).

Postoperatively, her new allograft produced $1 \mathrm{~L}$ of urine (1.1 $\mathrm{mL} / \mathrm{kg} / \mathrm{hr})$ in the first $24 \mathrm{hrs}$, but this declined to $300 \mathrm{~mL}(0.2 \mathrm{~mL} /$ $\mathrm{kg} / \mathrm{hr}$ ) of urine output in POD \#2 and POD \#3 respectively. Serum creatinine preoperatively was $5.95 \mathrm{mg} / \mathrm{dL}$ and declined to $5.57 \mathrm{mg} /$ $\mathrm{dL}$ by postoperative day 1 , then rising to $6.28 \mathrm{mg} / \mathrm{dL}$ on postoperative day 2 and $6.44 \mathrm{mg} / \mathrm{dL}$ by postoperative day 3. Given this decline in allograft function, we obtained a renal ultrasound with Doppler, which demonstrated mildly elevated resistive indices in the main renal artery and arcuate arteries. This ultrasound, in addition to a Mag3 renal scan, demonstrated findings consistent with acute tubular necrosis. Given the delayed graft function, we chose to intensify the induction immunosuppression from basiliximab to alemtuzumab (which was according to our protocol), as well as initiating dialysis. Her intravascular volume exam prior to alemtuzumab infusion demonstrated fluid overload with bibasilar rales, oxygen saturation of $100 \%$ with $2 \mathrm{~L}$ via nasal cannula, and trace peripheral edema. Given the impression of only mild hypervolemia and the logistics of arranging for dialysis, alemtuzumab was given first, with plans for dialysis immediately afterwards.

Halfway through the alemtuzumab infusion, our patient rapidly developed increased work of breathing, diffuse end-expiratory wheezing, markedly worsened rales, tachypnea, acute desaturation to 83 percent (on $2 \mathrm{~L}$ of supplemental oxygen via nasal canula), tachycardia, and an acute drop in systolic pressure from 160 to $100 \mathrm{mmHg}$. She required Bi-level Positive Airway Pressure (BiPAP) and a chest x-ray showed pulmonary edema. The alemtuzumab infusion was halted and dialysis was initiated urgently for volume removal. Within one hour, her acute respiratory failure resolved and she became normotensive, never requiring vasopressor support. However, the presumed cytokine storm (cytokine levels were not measured) was accompanied by a prolongation of her delayed graft function, presumably by capillary leak causing intrarenal edema [5]. Her allograft function ultimately did recover sufficiently to be independent from renal replacement therapy.

\section{Case \#2}

Our second patient in this discussion is a 59-year-old Caucasian woman with ESRD due to FSGS, who had relevant medical comorbidities including essential hypertension, mild chronic systolic heart failure (LVEF 54\%), and moderate aortic stenosis, but no prior kidney transplants nor other significant prior surgical history. She was on peritoneal dialysis prior to presenting for her deceased donor kidney transplant (but anuric), with her final dialysis performed overnight the day prior to the transplantation course we discuss here. The kidney donor for this transplant was 55 years old, had a profile index (KDPI) of $60 \%$, was a standard criteria (SCD) donor who died due to cardiac death (DCD). There were no HLA-DR mismatches, but 1 HLA-A and 2 HLA-B mismatches. Panel reactive antibodies (PRA) by flow cytometry were $0 \%$ for both Class -I and Class -II. Calculated panel reactive antibody level (cPRA) was $0 \%$.

She underwent immunosuppressive induction in the operating room with $20 \mathrm{mg}$ of IV basiliximab given one hour following $500 \mathrm{mg}$ of IV methylprednisolone (which incidentally was $11.8 \mathrm{mg} / \mathrm{kg}$ in this particular patient). Kidney transplantation surgery was performed without any apparent immediate complications. Her induction with basiliximab was chosen by our center-specific protocol (as aforementioned).

Postoperatively, her new allograft produced nearly $1 \mathrm{~L}$ of urine $(0.8$ $\mathrm{mL} / \mathrm{kg} / \mathrm{hr})$ in the first $24 \mathrm{hrs}$, but this declined to $225 \mathrm{~mL}(0.2 \mathrm{~mL} / \mathrm{kg} / \mathrm{hr})$ of urine output by POD \#3. Serum creatinine preoperatively was 5.38 $\mathrm{mg} / \mathrm{dL}$ and declined to $5.18 \mathrm{mg} / \mathrm{dL}$ by postoperative day 1 , then rising to $3.42 \mathrm{mg} / \mathrm{dL}$ by postoperative day \#3. Given this decline in allograft function, we obtained a renal ultrasound with Doppler, but found nothing abnormal. This was followed by a Mag3 renal scan, however, which demonstrated findings consistent with acute tubular necrosis. Given the delayed graft function, we chose to intensify the induction immunosuppression from basiliximab to alemtuzumab (as per our protocol). However, especially given our experience with the patient in case \#1, we opted to dialyze for intravascular volume optimization prior to alemtuzumab infusion. Her intravascular volume exam prior to alemtuzumab infusion demonstrated clear lungs, oxygen saturation of $96-99 \%$ with $2 \mathrm{~L}$ via nasal cannula, and no peripheral edema.

One hour after the alemtuzumab infusion, our patient developed rapid acute desaturation and required an escalation to $10 \mathrm{~L}$ oxygen via non-rebreather mask. Prompt clinical evaluation revealed nothing other than the aforementioned acute desaturation. In this case, her intravascular euvolemia substantially blunted the potential clinical sequelae from what was presumably capillary leak - and her chest $\mathrm{x}$-ray showed only mild pulmonary edema. Within 15 minutes, her oxygen saturation recovered without any intervention. The transient and self-resolving nature of her pulmonary decompensation was inconsistent with acute heart failure and there was nothing to suggest aspiration. Unlike the patient in case \#1, she fortunately did not suffer any hemodynamic instability.

\section{Discussion}

Both patients developed rapid and acute decompensation that can be explained by cytokine release syndrome from the alemtuzumab infusion. While there is a paucity of good evidence on who will develop cytokine release syndrome due to the initial dose of alemtuzumab, especially in organ transplantation, the degree of this response is thought to be modest [4]. Nonetheless, the spectrum of severity is broad. Symptomatic manifestations of this reaction can vary along the spectrum of systemic inflammatory response, including fevers, malaise, 
rigors, diaphoresis, and hypotension potentially requiring vasopressor support, tachypnea, hypoxia, capillary leak, and gastrointestinal symptoms of nausea, vomiting, or diarrhea [6]. The sequelae can include vasodilatory shock, acute kidney injury, pulmonary edema, acute liver injury, stress-induced cardiomyopathy, and acute encephalopathy.

The mechanism of cytokine release syndrome from first-dose alemtuzumab has been demonstrated in vitro and ex vivo where it was determined that its stimulation of lymphocytes and monocytes triggered release of several cytokines, including interleukin (IL)-6, tumor necrosis factor (TNF) $\alpha$ and interferon (IFN) $\gamma$, all by ligating the FC portion on natural killer (NK) cells, without any evidence of compliment activation [7]. Highly elevated levels of the aforementioned cytokines have also been detected during clinical presentations of cytokine release syndrome in several human patients being treated with various T-lymphocyte-depleting therapies for cancer [8].

There is no evidence-based data to guide prevention of cytokine release syndrome from alemtuzumab, but diphenhydramine 50 $\mathrm{mg}$, acetaminophen $650 \mathrm{mg}$, and steroids are recommended in the package insert [6]. The corticosteroid dose given prior to the first dose of alemtuzumab may reduce the severity of cytokine release, especially when being used for delayed graft function. While the package insert for alemtuzumab recommends premedication with equivalent of $32 \mathrm{mg}$ of methylprednisolone ( $200 \mathrm{mg}$ of hydrocortisone) prior to infusion, a higher steroid dose of $125 \mathrm{mg}$ might be warranted in the setting of delayed graft function to offset the potential severe cytokine reaction, as illustrated in case \#2 where flash pulmonary edema occurred despite receiving $1 \mathrm{mg} / \mathrm{kg}$ of steroid infusion and ultrafiltration with hemodialysis prior to drug administration. While optimization of intravascular volume prior to the first alemtuzumab infusion is especially pertinent when the drug is given for delayed graft function, the second case (in which dialysis was performed prior to alemtuzumab infusion) demonstrates that treatment of volume overload prior to the drug administration does not eliminate the risk of clinical evident cytokine release syndrome. Intravascular volume overload in end stage kidney disease patients can be insidious in the days immediately following kidney transplantation. While the jugular venous pressure is often a useful surrogate for the intravascular volume status, its utility can be limited by body habitus and tricuspid valvular dysfunction. As is often seen in dialysis patients, rising blood pressure can represent intravascular hypervolemia.

\section{Conclusion}

Given the risk of pulmonary edema due to capillary leak from alemtuzumab, dialysis should be strongly considered prior to the first infusion when given in the setting of delayed graft function. However, given the nature of capillary leak from cytokine release syndrome, optimized intravascular status prior to the first-dose administration of alemtuzumab does not completely negate the risk. While a higher steroid dose could perhaps mitigate the risk from first-dose infusion, the true adequate dose is not well established in this context. The risk of cytokine release syndrome from alemtuzumab, particularly in its use for treatment of delayed graft function, is very similar to the risk seen with OKT3 - and close monitoring, along with effective preventive protocols should be established.

\section{Declarations}

The authors declare no conflicts of interest.

\section{Ethics approval and consent to participate}

Not applicable.

\section{Consent for publication}

Not applicable to this case report, which contains no identifiable information

\section{Availability of data and material}

Not applicable.

\section{Competing interests}

None of the authors have any financial or non-financial competing interests to declare.

\section{Funding}

None.

\section{Authors' contributions}

DJR: participated in research design, participated in writing of the paper, participated in the performance of the research

ASO: participated in research design, participated in writing of the paper, participated in the performance of the research

DJN: participated in the performance of the research

SR: participated in the performance of the research

JBL: participated in research design, participated in writing of the paper, participated in the performance of the research

\section{Acknowledgement}

None.

\section{References}

1. Hale G, Bright S, Chumbley G, Hoang T, Metcalf D, et al. (1983) Removal of T cells from bone marrow for transplantation: a monoclonal antilymphocyte antibody that fixes human complement. Blood 62: 873-882. [Crossref]

2. 3C Study Collaborative Group, Haynes R, Harden P, Judge P, Blackwell L, et al (2014) Alemtuzumab - based induction treatment versus basiliximab - based induction treatment in kidney transplantation. Lancet 384: 1684-1690. [Crossref]

3. Hanaway MJ, Woodle ES, Mulgaonkar S, Peddi VR, Kaufman DB, et al. (2011) Alemtuzumab induction in renal transplantation. $N$ Engl J Med 364: 1909-1919. [Crossref]

4. Morris PJ, Russell NK (2006) Alemtuzumab (Campath-1H): A Systematic Review in Organ Transplantation. Transplantation 81: 1361-1367. [Crossref]

5. Norman DJ, Chatenoud L, Cohen D, Goldman M, Shield CF (1993) Consensus statement regarding OKT3-induced cytokine-release syndrome and human antimouse antibodies. Transplant Proc 25: 89-92. [Crossref]

6. Campath (alemtuzumab) (2003) Manufactured by: Millennium and ILEX Partners, LP Cambridge pp: 1-14.

7. Wing MG, Moreau T, Greenwood J, Smith RM, Hale G, et al. (1996) Mechanism of first-dose cytokine release syndrome by CAMPATH 1-H: involvement of CD16 (FcgammaRIII) and CD11a/CD18 (LFA-1) on NK cells. J Clin Invest 98: 2819-2826. [Crossref]

8. Lee DW, Gardener R, Porter DL, Louis CU, Ahmed N, et al. (2014) Current concepts in the diagnosis and management of cytokine release syndrome. Blood 124: 188-195. [Crossref]

Copyright: (C2020 Roberts DJ. This is an open-access article distributed under the terms of the Creative Commons Attribution License, which permits unrestricted use, distribution, and reproduction in any medium, provided the original author and source are credited. 